\title{
Mevalonic Aciduria as a Differential Diagnosis of Blueberry Muffin Baby
}

Rafaela Wagner ${ }^{1}$, Camila Vieira Bellettini ${ }^{1^{\star}}$, Márcia Bandeira ${ }^{2}$, Eduardo Maranhão Gubert ${ }^{1}$ and Mara Lúcia Schmitz Ferreira Santos ${ }^{4}$

${ }^{1}$ Department of Pediatrics, Hospital Pequeno Príncipe, Brazil

${ }^{2}$ Department of Pediatric Rheumatology, Hospital Pequeno Príncipe, Brazil

${ }^{3}$ Department of Pediatric Neurology, Hospital Pequeno Príncipe, Brazil

*Corresponding author: Camila Vieira Bellettini, Pediatric Medical Resident, Hospital Pequeno Príncipe, Brazil, Tel: 413310-1010; E-mail: camila.bellettini@gmail.com Received date: June 03, 2016; Accepted date: July 22, 2016; Published date: July 27, 2016

Copyright: (c) 2016 Wagner R, et al. This is an open-access article distributed under the terms of the Creative Commons Attribution License, which permits unrestricted use, distribution, and reproduction in any medium, provided the original author and source are credited.

\begin{abstract}
Mevalonate kinase (MK) deficiency is an auto inflammatory autosomal recessive disorder caused by a mutation in the gene encoding MK, an essential enzyme in the biosynthesis of cholesterol and isoprenoids. The disease has a wide clinical spectrum according to enzyme residual activity, the most severe form is known as Mevalonic Aciduria (MA). The aim of this article is to report a clinical case of severe MA, with an initial manifestation of a skin rash known as blueberry muffin, most commonly associated with congenital infections or neonatal hematopoietic diseases. We report the case of a three-month boy, born to consanguineous parents, that was referred to our service for assessment of malnutrition, vomiting, fever, jaundice and hepatosplenomegaly. At birth, he presented a purpuric rash suggestive of Blueberry Muffin Baby Syndrome. A thorough investigation revealed increased excretion of urinary mevalonic acid and a single mutation in homozygosis in exon 10 of the MK gene, which confirmed the diagnosis of MA. Our clinical case brings out the first report that associates blueberry muffin rash and MA.
\end{abstract}

Keywords: Mevalonic aciduria; Blueberry muffin baby syndrome

\section{Introduction}

Mevalonate kinase (MK) deficiency is an auto inflammatory autosomal recessive disorder caused by a mutation in the gene encoding MK, an essential enzyme in the biosynthesis of cholesterol and isoprenoids [1-5]. It catalyzes the conversion of mevalonic acid to mevalonate 5-phosphate, then the MK deficiency leads to increased serum, tissue and urine levels of mevalonic acid $[4,5]$.

The disease has a wide clinical spectrum according to enzyme residual activity, varying from recurrent febrile attacks, known as Periodic Fever Syndrome or Hyperimmunoglobulinemia D. To a more severe form, known as Mevalonic Aciduria (MA), which is characterized by failure to thrive, psychomotor retardation, cataracts, ataxia, dysmorphia and often early death $[3,4]$.

The aim of this article is to report a clinical case of severe MA, in which the first manifestation was a skin lesion known as Blueberry Muffin Baby Syndrome, most commonly associated with congenital infections or neonatal hematopoietic diseases.

\section{Case Report}

A three-month old boy, born to consanguineous parents, was referred to our service in order to investigate a progressive metabolic disease, after initial assessment and subsequent clinical follow-up resulted inconclusive.

At 36 weeks of gestation, an ultrasound demonstrated oligohydramnios, intrauterine growth restriction with an estimated fetal weight of $2933 \mathrm{~g}$, cardiomegaly, apparent hypertrophy of ventricular walls, and imminent signs of fetal distress.

An emergency C-section was performed and the patient was born steeped in meconium, with an APGAR of 8/9, Parkin evaluation of 39 weeks and $2970 \mathrm{~g}$ of weight. Physical examination revealed a maculopapular purpuric rash, with diffuse skin lesions, suggestive of Blueberry Muffin Baby Syndrome (Figure 1).

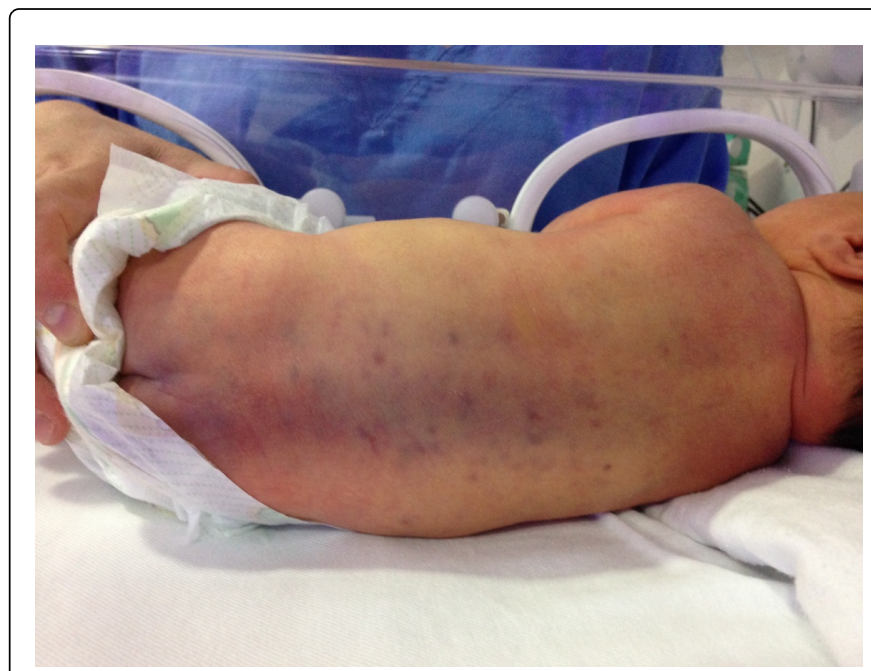

Figure 1: Blueberry muffin rash.

The new-born was immediately admitted to Neonatal Intensive Care Unit (NICU) for monitoring and further investigation of cutaneous lesions.

Laboratory tests performed at NICU admission revealed a leukemoid reaction with a leukocyte count of $89830 / \mathrm{mm}^{3}(15 \%$ lymphocytes, $11 \%$ monocytes, $74 \%$ neutrophils), thrombocytopenia $\left(79000 / \mathrm{mm}^{3}\right)$, direct hyperbilirubinemia $(9,03 \mathrm{mg} / \mathrm{dL})$, hypoglycemia $(20 \mathrm{mg} / \mathrm{dL})$, increase in C-reactive protein $(142,90 \mathrm{mg} / \mathrm{L})$ and lactate dehydrogenase (19066 U/L). Blood cultures were negative, as well as 
congenital infection tests (rubella, toxoplasmosis, human immunodeficiency virus, cytomegalovirus, herpes and Epstein-Barr virus).

The patient received antibiotic treatment with ampicillin and gentamicin in the first ten days of life. Due to the patient's critical state, skin biopsy was delayed. By the end of treatment, and resolution of leukemoid reaction, all lesions were gone and no further assessment was possible.

He was then discharged from the NICU, and referred to follow-up in a high risk new-born unit, for complementary investigation.

At three months of age, the patient was referred to our service because of persisting vomiting after feedings, high spiking and recurrent fever $\left(39-40^{\circ} \mathrm{C}\right)$ and worsening jaundice. The patient, who was discharged with minimal jaundice from de NICU, was admitted with direct bilirubin levels of $12 \mathrm{mg} / \mathrm{dl}$. On physical examination, the patient was in poor general condition, malnourished, pale, dehydrated, tachydyspneic, feverish, with cold extremities, weak pulses, distended abdomen, hepatosplenomegaly and multiple petechial lesions. The subject developed hemodynamic instability, requiring admission in ICU and institution of vasoactive drugs and parenteral antibiotic therapy for clinical stabilization. Laboratorial tests in the occasion revealed the maintenance of initial findings with increased liver enzymes, severe anemia (hemoglobin 6,0 g/dL), decreased albumin and abnormal coagulation tests.

Following the workup, an extended virological panel was performed and acute infections by Parvovirus B19, Hepatitis A, B and C were discarded. Bone marrow biopsy was also normal, discarding a neoplastic disease as well as a hemophagocytic syndrome.

Following the suspicion of a metabolic disease, the patient underwent dosages of alpha-fetoprotein, alpha-1-antitrypsin, amino acid chromatography in plasma, acyl carnitine profile and dosages of urinary organic acids, which revealed an increased excretion of urinary mevalonic acid $(3712 \mathrm{mmol} / \mathrm{mol}$ creatinine - reference value $<11.4$ $\mathrm{mmol} / \mathrm{mol}$ ). In the occasion, patient's serum total cholesterol was 170 $\mathrm{mg} / \mathrm{dl}$; triglycerides $198 \mathrm{mg} / \mathrm{dl}$; LDL $107 \mathrm{mg} / \mathrm{dl}$; HDL $23 \mathrm{mg} / \mathrm{dL}$, reveling a clear dyslipidemia profile, according to reference values for age.

Dosages of immunoglobulin E $(2308 \mathrm{UI} / \mathrm{mL})$ and $\mathrm{D}(247 \mathrm{U} / \mathrm{mL})$ were above the normal range. Immunoglobulin A ( $48 \mathrm{mg} / \mathrm{dL}), \mathrm{G}(1410$ $\mathrm{mg} / \mathrm{dL})$ and $\mathrm{M}(68 \mathrm{mg} / \mathrm{dL})$ had normal values.

Genetic sequencing demonstrated a single mutation in homozygosis in exon 10 of the MK gene variant c.893T>C (p.1298T), which confirmed the diagnosis of MA.

After diagnosis, the patient began treatment with canakinumab, a monoclonal antibody, with a very positive response. In the present date, clinical follow-up revealed an evolution of six months free form blood transfusions (hemoglobin of $10,4 \mathrm{~d} / \mathrm{dL}$ in the last control), no recurrence of fever, substantial neurological improvement, and normal liver function, with no jaundice.

\section{Discussion}

Mevalonic Aciduria (MA) is a rare autosomal recessive disease caused by a severe deficiency of mevalonate kinase (MK), with residual activity $<1 \%$. That results from a mutation in the gene encoding MK, an essential enzyme in the biosynthesis of cholesterol and isoprenoids. [1-5]. Auto inflammatory characteristic of MA seems to be associated with the fact that some isoprenoids work as negative regulators of inflammasome, a cytosolic multiprotein complex that generates the active forms of caspase-1, IL-1b, IL-18 and IL-33 [1].

In the past years, there was a significant increase in the understanding of the auto inflammatory syndromes. Despite of this progress, several issues about pathophysiologic mechanisms and treatments remain uncertain [1].

MA is a rare and severe disease. The early-onset version of the disease has poor prognosis: about half of patients do not reach adolescence [2]. It consists in a disorder with multiple organ involvement that may be associated with severe and/or recurrent bacterial infections, autoimmune cytopenia and macrophage activation syndrome [3].

Diagnosis is established by the detection of elevated excretion of mevalonic acid in urine and is confirmed by demonstration of deficient MK enzyme activity or by identification of two diseasecausing mutations in the MK gene [2]. High levels of immunoglobulin $\mathrm{D}(\mathrm{IgD})$ are characteristic, but are apparently not associated with the severity of pathophysiology of the condition $[6,7]$.

There is no established therapeutic regime for patients with MA. Most of the usual treatments, such as corticosteroids, NSAIDs and colchicine are not effective. The involvement of MK in the cholesterol synthesis pathway has encouraged the introduction of statins in the management of MA; some reports have demonstrated its efficacy, but it remains controversial $[2,4,6,8]$. The use of Anakinra, an interleukin-1-receptor antagonist, and tumor necrosis factor a (TNFa) antagonists (e.g. etanercept) has also been reported with some degree of success $[1,4,6]$, as well as monoclonal antibodies and bone marrow transplantation $[9,10]$.

The blueberry muffin rash is characterized by diffuse blue to purple, sometimes purpuric macules, papules or nodular lesions, with a dominant distribution in trunk, neck and head. It represents a potentially life-threatening condition, which historically is associated with congenital viral infections such as rubella and cytomegalovirus, hematologic dyscrasias or extramedullary hematopoiesis [11]. The differential diagnosis should include several neoplastic disorders, such as neuroblastoma, acute myeloid leukemia and Langerhans cell histiocytosis. As well as vascular disorders, such as hemangiomas, multifocal lymphangioendotheliomatosis and glomangioma. Neonatal lupus should also be considered in particularly ill new-borns, with a maternal history of erythematous systemic lupus, presenting with a diffuse rash [9].

All infants with multifocal blue papules or nodules should undergo a skin biopsy when possible, because the cellular composition of the lesion will determine further diagnostic evaluation. Many of the congenital cutaneous lesions may resolve spontaneously during the first days to weeks of life, therefore, prompt recognition and diagnosis is needed [12].

Our clinical case highlights the first report of association between blueberry muffin rash and MA.

\section{References}

1. Ruiz Gomez A, Couce ML, Garcia-Villoria J, Torres A, Baña Souto A, et al. (2012) Clinical, genetic and therapeutic diversity in 2 patients with severe mevalonate kinase deficiency. Pediatrics 129: e535-539. 
Citation: Wagner R, Bellettini SV, Bandeira M, Gubert EM, Schmitz ML (2016) Mevalonic Aciduria as a Differential Diagnosis of Blueberry Muffin Baby. J Neonatal Biol 5: 225. doi:10.4172/2167-0897.1000225

Page 3 of 3

2. Haas D, Hoffmann GF (2006) Mevalonate kinase deficiencies: from mevalonic aciduria to hyperimmunoglobulinemia D syndrome. Orphanet J Rare Dis 1: 13.

3. Bader-Meunier B, Florkin B, Sibilia J, Acquaviva C, Hachulla E, et al. (2011) Mevalonate kinase deficiency: a survey of 50 patients. Pediatrics 128: e152-159.

4. Neven B, Valayannopoulos V, Quartier P, Blanche S, Prieur AM, et al. (2007) Allogeneic bone marrow transplantation in mevalonic aciduria. N Engl J Med 356: 2700-2703.

5. Stabile A, Compagnone A, Napodano S, Raffaele CG, Patti M, et al. (2013) Mevalonate kinase genotype in children with recurrent fevers and high serum IgD level. Rheumatol Int 33: 3039-3042.

6. Jesus AA, Oliveira JB, Hilário MO, Terreri MT, Fujihira E, et al. (2010) Pediatric hereditary autoinflammatory syndromes. J Pediatr (Rio J) 86: 353-366.

7. Ammouri W, Cuisset L, Rouaghe S, Rolland MO, Delpech M, et al. (2007) Diagnostic value of serum immunoglobulinaemia $\mathrm{D}$ level in patients with a clinical suspicion of hyper IgD syndrome. Rheumatology (Oxford) 46: 1597-1600.

8. van der Burgh R, Ter Haar NM, Boes ML, Frenkel J (2013) Mevalonate kinase deficiency, a metabolic autoinflammatory disease. Clin Immunol 147: 197-206.

9. Mehta V, Balachandran C, Lonikar V (2008) Blueberry muffin baby: A pictoral differential diagnosis. Dermatol Online J 14: 8.

10. Tsitsami E, Papadopoulou C, Speletas M (2013) A case of hyperimmunoglobulinemia syndrome successfully treated with canakinumab. Case Rep Rheumatol 2013: 795027.

11. Holland KE, Galbraith SS, Drolet BA (2005) Neonatal violaceous skin lesions: expanding the differential of the "blueberry muffin baby". Adv Dermatol 21: 153-192.

12. Romina P, Larralde M (2011) "Blueberry muffin baby." Dermatol pediatr latinoam 1: 6-15. 